\title{
Playing is sharing. La condivisione e il tempo libero nelle reti sociali di gioco
}

Playing is sharing. Sharing in social gaming networks

\section{Aurelio Castro}

\section{OpenEdition}

1 Journals

Edizione digitale

URL: http://journals.openedition.org/aam/1442

DOI: $10.4000 /$ aam. 1442

ISSN: 2038-3215

Editore

Dipartimento Culture e Società - Università di Palermo

Notizia bibliografica digitale

Aurelio Castro, «Playing is sharing. La condivisione e il tempo libero nelle reti sociali di gioco », Archivio antropologico mediterraneo [Online], Anno XXII, n. 21 (1) | 2019, online dal 30 juin 2019, consultato il 23 septembre 2019. URL : http://journals.openedition.org/aam/1442 ; DOI : 10.4000/aam. 1442

Questo documento è stato generato automaticamente il 23 septembre 2019

Archivio antropologico mediterraneo 


\title{
Playing is sharing. La condivisione e il tempo libero nelle reti sociali di gioco
}

Playing is sharing. Sharing in social gaming networks

\author{
Aurelio Castro
}

\section{Introduzione}

1 Giocare è un'azione onnipresente nell'esperienza umana e le attività delle società umane sono «intessute di gioco» (Huizinga 2002: 3) e per un numero crescente di persone, di ogni età, dedicare tempo al gioco è un aspetto rilevante della propria identità, delle relazioni interpersonali e del proprio tempo libero. Dedicare tempo al gioco permette di mettersi alla prova, conoscere nuove persone, divertirsi, creare reti di socialità e spazi di partecipazione, identificarsi in un gruppo sociale e, infine, generare momenti di condivisione e di consumo consapevole. La condivisione del tempo di giochi e del giocare è una pratica presente da molti anni nei contesti ludici, sia in Italia che a livello internazionale, ed è un aspetto sociale poco approfondito in sociologia, la quale si è principalmente concentrata su altri temi: analizzare i giochi presenti nelle società, impiegare il gioco come strumento di analisi del comportamento, come metafora sociale ed economica o esperimenti di laboratorio sulla cooperazione e dilemmi etici (per approfondire si rimanda a Swedberg 2001: 309). Nonostante i contributi filosofici (Wittgenstein 1969'1) e antropologici (Turner 1982; Geertz 2000) allo studio dei giochi e del concetto di gioco siano stati fondamentali nello sviluppo della ludologia, spesso in altre discipline l'uso di giochi e modalità ludiche viene relegato agli studi o interventi psicologici sull'infanzia (Oren 2008; O'Connor et al. 2015: 46), a contesti formali di apprendimento (Gee 2003; Egenfeldt-Nielsen 2006) o a ricerche di mercato (Zichermann 2013; Lieberoth 2015). Pur mantenendo un costante interesse verso questi ultimi tipi di studi, recentemente si sta espandendo uno studio sociale e scientifico del gioco (Boellstorff 2006: 30) come ambito multidisciplinare focalizzato 
sugli aspetti ludici, creativi e trasformativi del giocare (Mochocki 2013; Karpatschof 2013; Daniau 2016).

2 Il presente saggio ha l'obiettivo di presentare una ricerca etnografica condotta tra diverse città italiane (Bologna, Catania, Padova e Torino) che ha esplorato tramite osservazione partecipante le potenzialità e modalità di attuazione del game sharing, ovvero la condivisione di giochi da tavolo e di ruolo da parte di associazioni ludiche, negozi e di gruppi di gioco indipendenti. La ricerca ha indagato come questi contesti di gioco permettano di creare una rete sociale basata sulla condivisione e su pratiche di consumo alternative. Inoltre, tramite interviste narrative semi strutturate (Riessmann 1993; Paolicchi 2002) sono state esplorate le narrazioni legate al tempo libero, al gioco e le interazioni tra players in modo da comprendere come, attraverso storie e pratiche, viene creata e ampliata una "cultura di gioco e del giocare", evidenziando sia le potenzialità (Daniau 2016) che le dinamiche sociali negative (Condis 2018) presenti in queste reti. In modo più ampio possiamo definire game sharing l'accesso condiviso a giochi da tavolo e di ruolo, processo che inizia generalmente nel momento in cui singole persone si organizzano per acquistare giochi da condividere tra loro e con chi sta iniziando o vuole iniziare a giocare. Alcuni di questi contesti privati maturano in associazioni culturali nel territorio che si espandono seguendo logiche non profit e gestiscono l'accesso alla condivisione e acquisto di giochi tramite votazioni ed elezione di referenti. Dall'altro lato, è anche possibile trovare singoli negozianti che decidono di mettere a disposizione, solitamente in orario extra-lavorativo, degli spazi per creare appuntamenti fissi, settimanali o mensili, in cui è possibile provare i loro prodotti iniziando quindi un processo di formazione del cliente prima del possibile acquisto. In generale, ciò che accomuna questi spazi, virtuali e fisici, è la passione per il gioco e il volerla condividere con altre persone sia nel proprio tempo libero che tramite la propria attività lavorativa.

\section{Cosa significa giocare e come studiare il gioco}

3 Nonostante il giocare e creare giochi siano attività socialmente condivise e presenti trasversalmente nelle società umane, definire in cosa consista un gioco (Boellstorff 2006: 30) non è un compito semplice. Diverse categorizzazioni e tassonomie sono state fornite nel corso del tempo (Huizinga 2002; Callois 2001; Arjoranta 2014: 4; Karpatschof 2013: 253) in diverse discipline, generalmente accompagnate a riflessioni sugli aspetti positivi o negativi del gioco (Chick 2015: 73). Per come inteso in questo saggio, possiamo definire come gioco un'attività condivisa basata su regole formali accettate da chi gioca e su regole informali "non dette", come l'accordo di fiducia sul non barare o sul completare un gioco che è stato iniziato. Il gioco è un'attività ricreativa libera, basata su partecipazione volontaria e divertimento, circoscritta in limiti di tempo e spazi, con esiti incerti nel corso della sua durata (Caillois, 2001: 4). Secondo Caillois (2001: 5) il gioco non è produttivo, ossia non ha come finalità quella di creare beni o ricchezze ulteriori rispetto a prima dell'inizio del gioco stesso; questa concezione viene però contestata, poiché si potrebbe affermare che l'esperienza stessa del giocare arricchisce chi gioca (Karpatschof 2013: 255; Fein et al. 2015: 300). Questa riflessione ci permette anche di distinguere il giocare dalle pratiche competitive e professionali di giochi, un dibattito recentemente aperto dagli sport elettronici, o e-sports, e dalla loro richiesta di riconoscimento in quanto sport al pari di quelli offline (Bányai et al. 2018: 
3). In generale, secondo alcuni studiosi (Caillois 2001) la competizione professionale e il ricavare benefici economici (esterni al gioco stesso) trasforma l'attività da ludica a lavorativa; invece, per altre posizioni (si veda Bányai et al., 2018: 10), il gioco competitivo può essere un'attività svolta "seriamente" nel proprio tempo libero, portando alcuni players a tentare una carriera professionale e altri solo a divertirsi mettendosi alla prova. Infine, nonostante sia bene mantenere definizioni inclusive di cosa significhi giocare e di cosa sia un gioco (Arjoranta 2014: 4), è importante chiarire che usare materiale di gioco, o legato ai giochi, non implica che in quel momento si stia "giocando" (Lieberoth 2015: 16) se l'obiettivo dell'interazione non è ludico (ad es. l'uso delle carte da gioco Dixit in psicoterapia come strumento proiettivo o impiegare una partita di Monopoly in un processo di selezione del personale).

4 Sia l'antropologia che la psicologia si stanno riavvicinando allo studio dei giochi sia online (Isabella 2007) che offline (Fein 2015) nelle società post-industriali tramite approcci di ricerca qualitativi, sperimentali e misti. Secondo Boellstorff (2006: 30), le potenzialità di contribuire allo studio dei giochi e le pratiche ludiche possono essere molteplici in quanto sia l'antropologia che la ludologia condividono l'interesse verso il significato, l'indicalità e la significazione. Concetti cruciali all'interno della performance di gioco e per lo studio del giocare. Dall'altro lato, i game studies possono trarre un grande beneficio dalla visione antropologica della cultura basata su relazioni, regole e costruzioni (Boellstorff 2006: 30) in contrapposizione a un uso sempre crescente di modalità simil-ludiche, definite come gamification (Zichermann 2013) e derivate dal marketing e da studi sui comportamenti di consumo (ad es. raccolta di punti o bollini, mettere in competizione i consumatori). Un approccio, questo, pesantemente criticato sia da chi fa ricerca (Lieberoth 2015: 16; Kapell 2015) che da esperti del settore ludico (Chick 2015: 73), poiché considerata una visione monetizzata e superficiale del potenziale del giocare (Mochocki 2013) sia dalle gaming communities che da chi sviluppa metodologie propriamente ludiche.

Infine, il concetto di gioco inteso nel presente contributo si distacca da ciò che vengono comunemente definiti come "serious games". Con questo termine ci si riferisce a dei giochi utilizzati sia per intrattenere che educare chi sta giocando su un tema specifico, come per esempio il cambiamento climatico (Reckien et al. 2013: 255). Usati spesso insieme ai serious games troviamo anche le simulations o dilemmi (ad es. il famoso dilemma del carrello ${ }^{2}$ ) in cui viene presentato un dilemma e/o una situazione da risolvere/analizzare che sarà utilizzata per valutarne le capacità di ragionamento o morali di chi porta a termine la prova. Le tipologie qui descritte sono distinte dai giochi, poiché nel "gioco" è fondamentale la componente di intrattenimento, che secondo alcuni è carente nei serious games, e ha degli obiettivi da raggiungere a differenza delle simulazioni (Egenfeldt-Nielsen 2006: 187). Infine, questo saggio non esplorerà tematiche legate alla ludopatia nelle pratiche definite "giochi d'azzardo" o i processi psicologici legati all'online video gaming (si rimanda a Markey et al. 2015) in quanto distinti dalle tipologie di giochi, da tavolo e di ruolo, più assiduamente praticate nei contesti di game sharing. Ricerche passate e recenti hanno indagato possibili parallelismi tra online gaming e gambling per gli sport elettronici (si veda Bányai et al., 2018), generalmente indagando le connessioni tra il giocare d'azzardo con il gioco ossessivo, tendenza al rischio, comportamenti di consumo e l'uso di videogame competitivi (ad es., pagare microtransazioni in denaro per avere premi casuali in gioco o acquistare vantaggi in gioco pagando somme di denaro). 


\section{La condivisione tra consumo e gioco}

Nella sua accezione più generale si usa il termine sharing quando è presente, tra singole persone e/o gruppi, una condivisione di spazi, di luoghi, di risorse o anche di competenze ed esperienze che siano normate da principi e convenzioni di condivisione. Uno dei principali settori in cui si è iniziato a parlare di sharing è l'ambito economico e del consumo collaborativo (Belk 2009), dove tramite la condivisione, online e/o offline, ci si propone come alternativa a servizi e canali tradizionali (Lunghi 2017: 67). Le opportunità economiche di chi ne usufruisce sono spesso considerate le principali motivazioni del successo della sharing economy (Schor 2014); poiché, grazie alla possibilità di risparmiare o di mettere a frutto le proprie risorse, queste occasioni sono attivamente ricercate dai diversi attori sociali che, spesso, rendendo meno netto il confine tra consumatore e produttore (Lunghi 2017: 68). Parallelamente alle motivazioni economiche, il fare uso di piattaforme o pratiche di sharing economy ha nel tempo contribuito a generare in chi ne usufruisce una forte componente di status sociale e, potenzialmente, anche appartenenza identitaria basata sulle pratiche di consumo non tradizionali. Oltre alle motivazioni economiche possiamo quindi trovare anche forti motivazioni ideologiche/politiche all'interno di questi contesti, non esenti dal rischio che questi ideali vengano compromessi. Visioni più critiche mettono infatti in guardia sulle potenzialità di profitto e di illegalità che alcune modalità della sharing economy possono offrire quando è carente la legislazione in materia; o quando il profitto possa attirare l'interesse di compagnie e aziende "tradizionali" (per un approfondimento si rimanda a Lunghi 2017).

7 Nel game sharing vengono resi accessibili e condivisi giochi da tavolo, di ruolo, di carte collezionabili e non. Il termine sharing è qui usato per riferirsi alla messa in condivisione, anche se è bene chiarire che l'accessibilità varia nei diversi contesti esplorati dalla ricerca a seconda delle situazioni: i negozi che praticano game sharing non chiedono nessuna quota a chi gioca (in un solo caso è stato proposto a chi scrive di tesserarsi per avere accesso a prenotazioni e sconti). Le associazioni culturali osservate durante la ricerca chiedono ai soci una quota quando si partecipa a una serata di gioco, la quota varia da associazione e città con una media di $2.5 €$ per giocare tutto il giorno o la sera. Infine, i gruppi di gioco indipendenti che ho incontrato gestiscono l'acquisto di giochi a rotazione tra i diversi membri.

Riguardo le abitudini di acquisto, la rete sociale di gioco e il game sharing offrono a chi gioca o a chi si sta avvicinando al gaming un'occasione di confronto con persone appassionate e l'opportunità di effettuare un acquisto consapevole, sia avendo modo di provare i prodotti che più suscitano interesse, sia avendo accesso a una consulenza esperta da parte di personale, giocatori e giocatrici esperti. Generalmente, il prezzo di un gioco da tavolo parte da $20 €$ per la maggior parte dei party game (giochi definiti come più semplici e adatti a gruppi numerosi) arrivando a un prezzo medio di $35 €$, mentre per alcuni giochi il prezzo può salire fino agli $80 €$ o ai $120 €$ in caso di giochi strategici a lunga durata e ricchi di componenti (ad es. miniature, cartonati, elementi in vetro). La maggior parte delle associazioni di game sharing acquistano mensilmente, o nel corso dell'anno, nuovi giochi da poter provare nelle loro sedi, arrivando ad accumulare nel corso degli anni molti giochi (più di 150 giochi da tavolo a Catania 
acquisiti in 7 anni; un'associazione di Bologna ha più di 60 giochi accumulati in 4 anni, di cui molti ricevuti come donazioni) anche se non tutti assiduamente giocati.

\section{Cornice teorica della ricerca}

Lo studio dei giochi, o ludologia, non ha un framework teorico e metodologico predefinito, si arricchisce sia di singoli contributi sia grazie al confronto con le scienze sociali, l'informatica, la filosofia (Karpatschof 2013: 255), le discipline artistiche e umanistiche. Adottando un approccio interdisciplinare tra psicologia sociale e antropologia culturale (Mantovani 1998), la ricerca si focalizza su «come le persone dicono che è il proprio mondo» (Bruner 1990; Paolicchi 2002: 196) e le modalità con cui viene messo in scena (Simon et al. 2003: 493). L'approccio etnografico ha permesso di esplorare e comprendere come pratiche sociali e significati legati al tempo libero e al gioco prendono forma in spazi di gioco condivisi. La prospettiva narrativa ha reso possibile indagare l'intreccio tra azioni sempre individuali, di giocatori e giocatrici, e sistemi di riferimento sempre culturalmente definiti (Paolicchi 2002: 196). La letteratura si è inoltre concentrata sul significato del gioco per come esso scaturisce dal processo di tensione dialettica tra gli strumenti culturali della collettività e dall'uso che di essi ne fa l'individuo (Bruner 1990; Riesmann, 1993; Paolicchi 2002: 196).

\section{Metodologie}

10 Durante la ricerca sono state impiegate diverse metodologie per comprendere i punti di incontro tra le pratiche performative legate al gioco e le narrazioni di chi gioca. Nella prima fase è stata condotta un'etnografia tramite molteplici osservazioni partecipanti, da maggio 2017 a giugno 2018, presso le sedi di associazioni di gioco e negozi che attuano game sharing. Degli spazi associativi osservati, uno è in affitto (Catania) mentre l'altro viene attualmente condiviso con altre associazioni (Bologna); dei negozi osservati solo uno è di proprietà (Padova) mentre i restanti sono in affitto (Torino, Bologna). Inoltre, tramite la rete di conoscenze acquisite in queste osservazioni è stato possibile accedere a degli eventi per "creatori di giochi" (per una riflessione sulla declinazione maschile di questi eventi si vedano i risultati) emergenti o con prototipi di gioco in fase di sviluppo. In queste osservazioni "coperte" (ossia non dichiarate), usando una cover story come appassionato di giochi, sono stati osservati i processi di significazione e le rappresentazioni del giocare da parte di chi vuole o sta creando giochi, oltre a delineare il copione delle fasi di sviluppo di un gioco da tavolo. Nel corso delle etnografie aperte e coperte la maggior parte delle note di campo sono state prese prima dell'ingresso al campo e subito dopo. Durante alcune sessioni di gioco è stato possibile prendere appunti, quando il gioco stesso permetteva di segnare punteggi o altre informazioni rilevanti.

11 Successivamente, sono state condotte delle interviste narrative (Riesmann 1993; Paolicchi 2002: 196) semi-strutturate (6 a Catania, 4 a Padova) in modo da accogliere le storie di chi gioca e comprendere, tramite le loro esperienze, come ci si appassiona ai giochi, come si sviluppano preferenze verso particolari tipologie e stili (competitivo, cooperativo, misto) quali sono le sensazioni ricercate, che tipo di relazioni hanno all'esterno della rete di game sharing, quanto tempo dedicano al gioco e quali pratiche di acquisto e consumo mettono in atto. Inoltre, sono state condotte ulteriori interviste 
libere con esperti di settore: due creatori di giochi (Torino, San Benedetto del Tronto), un creatore di giochi e membro di un'associazione di gioco LGBT+ (Bologna), un dimostratore di giochi (Bologna), una ricercatrice indipendente su temi di genere, orientamento sessuale e gioco (Treviso). Le interviste sono state condotte di persona eccetto due, una condotta telefonicamente con un dimostratore di giochi (Bologna) e una via Skype (Treviso). Una volta trascritte le interviste sono state codificate in modo aperto concentrandosi sul processo di creazione della storia (Riessman 1993), i "punti di svolta" nella narrazione, i significati associati al gioco e infine i contenuti di potenziali "copioni" (Simon et al. 2003: 493); le interviste sono state integrate con le note di campo e, quando possibile, chiedendo chiarimenti alle persone intervistate o osservate. Nei prossimi paragrafi saranno presentate le tematiche principali emerse dal lavoro di ricerca: il rapporto tra rete sociale e tempo libero, lo scambio tra reti di gioco e mondo del lavoro e, infine, le forme di resistenza sociale verso i pregiudizi e le discriminazioni presenti all'interno delle gaming communities.

\section{Reti sociali di gioco e tempo libero}

Dall'analisi etnografica e dalle interviste emergono in particolare due temi: la costruzione e l'ampliamento della rete sociale di gioco e l'affermazione del gioco come attività anche adulta per il proprio tempo libero. Con rete sociale si intende qui l'insieme interdipendente di persone, gruppi sociali, legami, conoscenze, associazioni, istituzioni e/o luoghi di riferimento significativi in determinati e specifici contesti o sottoculture (Leone et al. 2013). Chi frequenta associazioni di game sharing entra a far parte di reti sociali ampliate basate sul gioco che coinvolgono, con diverse gradazioni, persone che giocano in modo assiduo (definiti spesso come core players), persone che giocano saltuariamente (casual players) e persone che si stanno avvicinando al gioco (spesso definiti col termine newbies); categorizzazioni costruite all'interno delle stesse community di gioco per demarcare (con effetti anche negativi come discusso più avanti) il grado di competenza di chi gioca, spesso a prescindere dal reale grado di competenza o abilità di gioco. Inoltre, si aggiungono agli attori e alle attrici sociali incontrati di persona in queste reti le più ampie community online costituite da piattaforme di gioco, social networks e siti di settore dove acquistare o leggere recensioni sui giochi. L'insieme di queste reti offline e online, continuamente in espansione, rende estremamente complesso mapparle dettagliatamente. Anche se le reti online sono in aumento, vengono principalmente impiegate per trovare gruppi e associazioni di gioco sul territorio o, nel caso non ce ne siano, di organizzarsi per crearne di nuovi. Una strategia legata anche alla fisicità del gioco da tavolo come espresso da un giocatore padovano (Uomo 26 anni): «Dal vivo alla fine ti diverti di più, soprattutto se ci sono le pedine o le miniature tocchi con mano il gioco. Ti fanno apprezzare di più il gioco perché alla fine lo paghi tanto anche per quello, e poi quando attacchi le miniature degli altri giocatori li guardi in faccia. Online non lo puoi fare». Che si adottino stili competitivi o cooperativi, il gioco da tavolo sembra essere apprezzato maggiormente dal vivo, soprattutto se giocato con players al proprio "livello" e non casualmente online. Anche se il gioco da tavolo offre l'occasione di giocare con persone di diversi livelli di esperienza, mantenendo la sua capacità di intrattenimento e sfida, nel corso delle osservazioni sono emerse opinioni contrastanti: nonostante giocatori e giocatrici esperti raccontano di poter divertirsi giocando con persone meno esperte, a lungo andare il non avere occasioni di sfidare persone di pari 
livello può portare a frustrazioni (Khan et al. 2015) o a isolarsi in gruppi minori formati da altre persone ritenute esperte o core players.

L'aspetto del promuovere la passione e la cultura di gioco è una costante nelle narrazioni di chi gioca e di chi crea giochi. Diffondere una prospettiva del gioco come una valida forma di tempo libero adatta a persone di ogni età è uno degli obiettivi principali delle associazioni e dei contesti osservati, sia nella ricerca che nelle community online. Il modello più adottato dalle associazioni di game sharing per fornire occasioni di promozione culturale consiste nel creare uno spazio condiviso dove poter giocare con una cadenza fissa (più volte a settimana, ogni settimana o ogni mese) e proporsi come spazio sul territorio per espandere la propria rete di giocatori e giocatrici. Successivamente, le associazioni cercano di avviare progetti scolastici per portare il gioco nelle scuole e provare nuove strategie di apprendimento (Baid et al. 2010: 550) e anche eventi pubblici legati al gioco. Un altro canale di promozione per la cultura di gioco sono quelli fieristici: eventi su larga scala dedicati a fumetti, giochi da tavolo e di ruolo, videogame e cultura "nerd"

Promuovere la cultura del gioco è rilevante anche per chi ha attività lavorative legate ad esso, incrociando motivazioni sia di profitto che di passione personale. I negozianti intervistati nel corso della ricerca riaffermano anche come sia stata la voglia di giocare a spingerli ad aprire queste attività, affrontando molte difficoltà in quanto attività di nicchia con elevati costi e pochi ricavi. Un partecipante (Catania, uomo, 32 anni) afferma che la dimensione ludica rimane presente anche per chi lavora in questo settore (ad es. mentre spiega i giochi ai clienti) anche se gli obiettivi sono differenti e non connessi soltanto alla fidelizzazione del cliente. Sembra invece esservi un tentativo di promuovere la cultura del gioco:

Guarda è un pub come gli altri ma abbiamo questa cosa in più, il ludopub lo senti come una cosa nuova. C'è la voglia di far crescere le persone, di far apprezzare il gioco. Anche perché, parlando dal punto di vista meramente economico se fai giocare un gioco male magari non ti diverti e non torni. Poi a me di questo non mi interessa [l'aspetto economico] ...io voglio che le persone giochino i giochi per quello che sono semplicemente perché io sono un appassionato di giochi. E voglio che questa cultura del gioco esca.

L'ampia offerta di giochi è funzionale alle occasioni di condivisione e di vendita. Come discusso da un negoziante e creatore di giochi (Torino, Uomo 45 anni) spesso questo mondo è sconosciuto ed è molto complesso da seguire dato il numero di uscite: «Mi chiedono "sono nuovi questi giochi?". [...] no, escono 3000 giochi nuovi all'anno almeno in Italia. Tra riedizioni, espansioni e giochi identici mascherati, non è tutto oro ciò che luccica. Di giochi veramente importanti un centinaio». Ciò nonostante, un mercato così ampio permette facilmente di trovare un gioco adatto a qualsiasi situazione e audience, da proporre alla propria rete di gioco o in famiglia. Giocatori e giocatrici, esperti o meno, riferiscono di passare più tempo libero con le proprie famiglie e amici impegnati in giochi da tavolo e, a volte, di ruolo. Attività viste inizialmente con diffidenza rispetto a giochi più "classici", ma in seguito accettate e preferite poiché favoriscono uno scambio comunicativo e competizione, spesso, positiva (Zagal 2006). Come riportato da due intervistati catanesi (Donna 35 anni e Uomo 55 anni): «Adesso la festività a casa nostra significa fare giochi da tavolo invece della classica briscola o tombola. [...] All'inizio ci guardavano straniti chiedendoci perché giocassimo a cose da ragazzini, ci è voluto del tempo ma dopo che hanno giocato si sono appassionati». 


\section{La rete di gioco tra scambio e lavoro}

16 Tramite le reti sociali, chi gioca non trova soltanto persone con cui condividere una passione e il proprio tempo libero ma anche possibilità lavorative e di sviluppo personale. Per molti partecipanti giocare è entrato a far parte della propria attività lavorativa, iniziata o trovata tramite le reti di gioco e del mondo ludico (ad es. aprire un negozio di giochi o una fumetteria, lavorare per un'azienda produttrice, progettare e creare nuovi giochi, lavorare come agente commerciale o come presentatore o presentatrice di giochi). In particolare, non sono emerse narrazioni in cui il tempo libero viene contrapposto al tempo dedicato al lavoro, sia per chi lavora in ambito ludico sia per chi svolge altre attività lavorative (Boellstorff 2006: 32; Karpatschof 2013). Nonostante il gioco sia spesso ridotto allo stereotipo di attività infantile, molte giocatrici e giocatori agiscono in modo da far entrare nel loro lavoro quotidiano aspetti e metafore del gioco anche per affrontarlo in modo propositivo e non cercando rifugio nel mondo di gioco (Zagal 2006; Calleja 2010). Per la maggior parte dei players osservati in etnografia o intervistati, al gioco viene dedicata la maggior parte del proprio tempo libero, occupando dalle 4 alle 10 ore settimanali su una media di 2-3 incontri settimanali dedicati al gioco. Per chi lavora in ambito ludico la gestione del tempo aumenta di complessità, spesso dedicando anche 15-20 ore fuori dall'orario di lavoro ad attività legate al gioco. Generalmente con una prospettiva di miglioramento dell'esperienza di gioco, propria o altrui, o di sviluppo personale e dei prototipi per chi crea giochi.

Il gioco di ruolo e da tavolo è anche un ottimo terreno di prova per sviluppare nuove abilità sociali e relazioni. Se gestiti positivamente e in modo costruttivo, i contesti di gioco rappresentano un campo protetto dove "mettere in scena" (Simon et al. 2003) nuove abilità, strategie di negoziazione e anche solo di apertura interpersonale (Gee 2003). I giochi, soprattutto quelli di ruolo, portano giocatrici e giocatori a mettersi nei panni delle altre persone al tavolo, a capire le strategie e le motivazioni delle loro scelte di gioco (Daniau 2016). Anche se ciò accade solo indicativamente per ottenere una "performance vincente", trasversalmente permette di facilitare i processi di assunzione di un ruolo e di provare empatia verso l'Alter (Mochocki 2013; Fein 2015). Un'occasione preziosa non solo a fini didattici ma anche per percorsi trasformativi personali (Daniau 2016), come riportato da un partecipante (Bologna, Uomo 26 anni) che riflette sul gioco di ruolo: «Se a un giocatore gli piace davvero giocare, accetta di vivere i panni di qualcun altro. [...] [giocare] ti fa sperimentare l'empatia. Che è una cosa buona da adolescente 9 volte su 10 , cioè se un adolescente una volta nella sua settimana sperimenta l'empatia da insegnante sarei contento».

Restando nelle potenzialità del giocare, è stato interessante notare come diversi partecipanti abbiano riportato esperienze simili nello sviluppo di soft skills grazie all'attività di gestione di tavoli da gioco. Sia durante le osservazioni a Catania che a Modena e Bologna ci sono stati momenti di confronto su come il ritrovarsi nel ruolo di "facilitatore" - ovvero quando si spiega un gioco a persone novizie - abbia avuto ripercussioni positive nella vita lavorativa dei partecipanti. A volte permettendo di ottenere offerte di lavoro, promozioni a ruoli di formazione o di team building. La performance della spiegazione e della mediazione richiede a chi se ne fa carico di sviluppare e allenare adeguate soft skills come l'ascolto attivo, adottare uno stile comunicativo chiaro e sintetico, coordinare gruppi di persone sconosciute e la 
costruzione di strategie condivise. Usando le parole di un dimostratore di giochi si può dire che «guidare gruppi di gioco mi ha permesso di guidare meglio il mio gruppo sul lavoro. Poiché io ero quello che imparava le regole e poi le spiegava», processo legato alla natura task oriented del gioco da tavolo e di ruolo, non dissimile alle mansioni svolte nella maggior parte delle attività lavorative di team building.

Infine, le reti di gioco offrono un'occasione di cooperazione e scambio sia di competenze che di servizi. Avere accesso a una rete estesa basata su una passione comune permette, a chi ne fa parte, di poter risolvere problematiche esterne al gioco stesso, come avere accesso a servizi professionali, consulenze e assistenza. Dalle diverse osservazioni e interviste è infatti emerso come si venga a creare una rete di saperi professionali trasversali all'interno sia delle associazioni che nelle comunità online di gioco. Queste reti diventano una delle prime fonti a cui chiedere aiuto professionale e ricercare competenze, sia direttamente (ad es. rivolgersi a membri dell'associazione che svolgono certe professioni) o indirettamente (ad es. chiedere se si conosce uno specifico professionista). In particolare, su 15 interviste svolte, più di metà dei partecipanti ha riportato di aver avuto modo di candidarsi a delle posizioni lavorative o di ricevere delle offerte di lavoro proprio grazie alla rete di gioco, sia in settori legati al mondo ludico sia in ambiti esterni al gioco.

\section{Pregiudizi, generi e giochi}

Le dimensioni del gioco e i suoi contesti non sono esenti da processi di prevaricazione, discriminazione e ostracizzazione (Condis 2018). Nel corso della ricerca è emerso come atteggiamenti negativi, pregiudizi e discriminazioni riguardassero principalmente il livello di esperienza (reale o percepita), il genere e l'orientamento sessuale di chi gioca. Una distinzione fondamentale emersa in questa etnografia, sia nella fase "coperta" sia in quella dichiarata, così come anche nelle interviste è la costruzione della dicotomia " core vs casual players", queste categorie rappresentano il grado di esperienza, dedizione e competenza che chi gioca incanala nell'attività ludica. Il casual player viene identificato come una persona che gioca occasionalmente e solo ai party game (a volte neanche considerati o definiti come veri giochi) mentre l'essere un core player è un ideale a cui aspirare poiché indica maggiore esperienza di gioco con sistemi di regole più complessi e dalla durata maggiore (definiti come "german" in quanto spesso prodotti in Germania). Dallo studio è emerso come il livello di esperienza di chi gioca possa diventare motivo, o più spesso pretesto, di esclusione dal tavolo di gioco oppure di allontanamento dall'associazione di giocatori e giocatrici autodefinitisi esperti che riferiscono di non trovare persone al proprio livello. Mentre alcune associazioni hanno optato per strategie di rafforzamento "chiuse", ovvero mantenere solo membri core e rendere più selettivo l'accesso, altrettante puntano su reclutamenti più ampi e costosi, spesso a costi personali. Le attività commerciali che praticano game sharing tendenzialmente preferiscono puntare sulla fidelizzazione di core player in quanto consumatori di giochi più costosi.

21 Il genere di chi gioca è la variabile principale quando vengono messi in scena stereotipi e discriminazioni verso donne e persone di genere non-binario nei contesti di gioco, sia online che offline (Condis 2018). Questa tematica è emersa sia tramite le interviste con una giocatrice, sia con un'esperta di settore di tematiche di genere e LGBT+. Sono infatti presenti delle forme di sessismo implicito in alcuni contesti di gioco che portano a 
percepire queste attività, ma anche a incorniciarle socialmente, come tipicamente maschili (Gray 2017). Il non essere uomo diventa motivo, o meglio pretesto, di delegittimazione delle proprie competenze e abilità di giocatrice, a prescindere dal reale grado di esperienza (Kishona et al. 2016). Soprattutto nei giochi di ragionamento, calcolo e strategia questa stereotipizzazione diventa più frequente, emergendo in modo preponderante in chi gioca da poco tempo o in chi appartiene a generazioni precedenti agli anni Ottanta. L'aver assistito a forme di tokenism ${ }^{4} \mathrm{e}$ al riferirsi all'attività dello sviluppare giochi come un "qualcosa che le mogli sopportano dei propri mariti" lascia intuire che implicitamente il gioco viene considerato un copione (Simon et al. 2003) maschile. In modo rilevante è emerso come spesso la presenza femminile al tavolo sia al massimo tollerata e vista con una doppia morale, per cui si vogliono più giocatrici ma pretendendo che seguano modelli maschili di gioco. Riflessione perfettamente sintetizzata da una intervistata:

«Le donne sì, ma nel modo giusto [...] I giocatori maschi si sono sempre lamentati della scarsa presenza femminile al tavolo di gioco, eppure quando le donne arrivano, si scatenano una serie di problematiche: mansplaining, discriminazione, mortificazione a vari livelli» (Donna 31 anni).

Tramite le etnografie coperte ho avuto modo di osservare degli atteggiamenti specifici verso giocatrici donne, a volte considerate come meno adatte a giochi da "esperti" e più a giochi casual da festa, i party game. Per esempio, durante una partita di playtest $^{5}$ con quattro giocatori, tutti uomini, uno di essi si esprime su delle potenziali giocatrici al tavolo mentre il creatore del gioco ribatte:

Giocatore: Già Coloni di Catan non è per tutti tutti. Eh, insomma [...] Ho giocato anche con molte ragazze e le ragazze specialmente con un gioco così, perdono l'attenzione dopo un po'. Non è un gioco da feeling per le ragazze, almeno secondo me $[. .$.$] con quelle con cui ho giocato, sono più da party game.$

Creatore di giochi: Le ragazze sono più attente alla gestione rispetto al combattimento, o agonismo. Sono più attente alla parte gestionale e alla parte di accumulo e gestione risorse.

Giocatore: Premesso che la maggior parte delle ragazze non è che giocano al tavolo per giocare.

Creatore di giochi: eh no invece [...] poi dipende, dipende dalle varie esperienze.

Colpisce in particolare la delegittimazione della presenza femminile al tavolo in quanto non realmente interessata al gioco e che ritiene come le donne non giochino per passione o abilità ma solo per altri motivi, per esempio il venir "trascinate" da un partner uomo (Gray 2017). Infine, quando atteggiamenti e pratiche tossiche sono presenti all'interno di una community emergono frequentemente discriminazioni verso sessualità e identità di genere non normative (Condis 2018) anche all'interno delle stesse comunità di gioco. Durante l'intervista con un creatore di giochi, e membro di un'associazione di gioco LGBT+ che pratica game sharing, sono emerse difficoltà di accettazione dell'intreccio tra queste due identità, sia nel mondo LGBT+ sia in quello "nerd". Come per altre intersezioni, le avversità incontrate riguardano la categorizzazione in etichette negative: alcune persone LGBT+ spesso vedono chi gioca come una persona infantile o "sfigata" poiché si dedica ad attività poco al passo coi tempi e al tempo stesso si viene discriminati da giocatori e giocatrici sessisti e antiLGBT per il proprio orientamento sessuale. Nonostante l'atteggiamento verso le persone con interessi "nerd" sia cambiato in positivo negli ultimi anni, entrando a tutti 
gli effetti nella cultura pop dominante, l'etichetta di "nerd e sfigato/a" resta ancora presente nell'immaginario.

Lo stesso partecipante riporta un episodio legato a un progetto di gioco finanziato tramite crowdfunding e le difficoltà incontrate nel trovare supporto nei gruppi social di gioco: «quando siamo andati su pagine Facebook a chiedere pubblicità alcune si sono rifiutate rispondendoci "bel progetto ma non me la sento"». Approfondendo questo episodio emerge il timore di perdere consenso se ci si schiera apertamente a favore di certe tematiche poiché una fetta di giocatori, soprattutto di generazioni precedenti agli anni ' 80 (Condis, 2018) hanno atteggiamenti molto negativi o apertamente ostili verso identità non eteronormative:

Si rischia, essendo il loro pubblico, a quanto ne sanno, totalmente etero che, dal loro punto di vista, si fa la parte di quelli... di quelli omosessuali. C'è paura di dire "io condivido le cose dei gay", perché chissà il pubblico come reagisce. Non hai mai condiviso un contenuto LGBT e, alla fine, dai per scontato di sapere ciò che la gente vuole o non vuole (uomo, 26 anni).

\section{Potenzialità del gioco e resistenza sociale}

Nel paragrafo precedente è stato chiarito come anche i contesti e le comunità di gioco non siano esenti da conflitti e discriminazioni. Allo stesso tempo, queste riflessioni hanno fatto emergere nel corso della ricerca come associazioni e singoli individui adottino strategie di resistenza per affrontare discriminazioni e integrare tematiche socialmente rilevanti nel corso del gioco. Una prima forma di inclusione sociale tramite il gioco nasce a livello interpersonale (Crisp, Turner 2009) quando l'entrare in contatto con soggettività diverse permette di riflettere su pregiudizi personali e dinamiche di genere, orientamento e grado di esperienza. Come riportato da un partecipante (Bologna, Uomo 26 anni) è soprattutto il gioco di ruolo ad offrire queste opportunità riportando che "cavalcando" il momento di condivisione offerto dal gioco possono nascere occasioni di apertura: «Con un sacco di persone ho avuto modo di relazionarmi, ho affrontato i primi passi nel parlare di me senza vergognarmi. Quanti coming-out a fine di sessioni ho fatto, quello è stato il lato più bello del gioco di ruolo nella mia adolescenza».

Trovarsi di fronte a un tavolo con delle persone e giocare può diventare un'occasione di riflessione interiore per entrare in contatto (Crisp, Turner 2009) con identità non normative con cui si condividono delle passioni e si scambiano storie (Fein 2015). La condivisione di storie permette di sostenere l'evolversi dell'immaginario collettivo creato durante l'evento, l'essere un gruppo che porta a termine delle missioni e si sostiene a vicenda, per poi ritornare al ruolo di giocatori da quello di personaggi tramite un approccio intersoggettivo (Daniau 2016). Questo rapporto viene valorizzato e accresciuto dall'investire tempo e risorse insieme (Crisp, Turner 2009) in un contesto che punta al confronto, alla competizione positiva e al gioco, non necessariamente allo stesso modo di un legame di amicizia o di conoscenza interpersonale. Come riporta ancora lo stesso creatore di giochi (Bologna, Uomo 26 anni): «Perché forse la cosa più importante che ho imparato, è riuscire a socializzare, ad avere un legame con persone che con me non c'entravano nulla». Questa forma di "scambio" può avvenire sia di persona che tramite piattaforme online ed è un punto di forza del promuovere interventi psicologici (Fein et al. 2015: 317), attività ludiche educative (Zagal 2010) e formative (Baid et al. 2010). Un processo sociale che passa anche dalle modalità di 
rappresentazione delle minoranze all'interno dei giochi, e in generale dalla richiesta di maggiore diversità in essi. Tematiche "calde" che non sempre suscitano reazioni positive nel pubblico (ad es. introdurre in un gioco un personaggio apertamente omosessuale o bisessuale) e portano a interazioni tossiche online (Condis 2018). Per promuovere questo tipo di riflessioni è necessario ottenere visibilità e un approccio non aggressivo, evidenziando che esistono disparità di rappresentazioni (Williams et al. 2009: 817), posizione espressa da un'esperta di settore su questioni di genere nel gaming:

«Intanto parlandone, che è lo scopo principale del gruppo. Non vogliamo, infatti, fare "didattica": dimostrare delle tesi, dare delle lezioni [...] Assolutamente no! Il nostro scopo è animare il dibattito, fornire dati concreti su cui articolarlo. E poi promuovendo il più possibile giochi che abbiano una prospettiva differente, inclusiva e rappresentativa delle minoranze» (Donna 31 anni).

\section{Riflessività e limiti della ricerca}

Lo studio esplorativo qui presentato non è esente da limiti e criticità. Anche se le osservazioni partecipanti di chi scrive sono state accompagnate da curiosità e interesse, bisogna considerare come l'essersi presentato da ricercatore, uomo cisgender ${ }^{6}$, possa aver influito sulle interazioni nei contesti di gioco, sulla conduzione delle interviste e la loro analisi. Essendo il genere una variabile rilevante per generare fenomeni di delegittimazioni e discriminazione in alcuni contesti tossici (Condis 2018), plausibilmente non è stato possibile esplorare in prima persona queste dinamiche in quanto ricercatore uomo. Per situare meglio la ricerca bisogna quindi riflettere su come la performance di ricerca sul gioco e il tempo libero sarebbe stata percepita se condotta da una ricercatrice donna o non binaria (Gray 2017). Oltre al genere, l'aver condotto la ricerca presentandosi come una persona non esperta di giochi potrebbe aver influito nell'interazione col campo, venendo percepito come un casual player invece che come un core player o le diverse intersezioni tra queste variabili precedentemente discusse.

Pur mantenendo un atteggiamento positivo nei confronti dei mondi di gioco, e di chi gioca, esplorare anche processi negativi e tossici presenti nelle comunità di gioco è un passo importante per i contesti di gioco stessi nel diventare più inclusivi e aperti a chiunque. Fermo restando che questi atteggiamenti tossici non sono strutturali al gioco da tavolo e di ruolo e che non hanno nulla in comune con la positiva competizione informale riscontrata all'interno di gruppi e associazioni esplorati. Alcune ricerche hanno argomentato come gli spazi virtuali di gioco, generalmente legati ai Massive Multiplayer Online, siano legati a processi psicologici negativi come escapismo (Calleja 2010) e atteggiamenti ossessivi (Lafrèniere 2010), ricerche future potrebbero esplorare processi simili nei contesti di gioco da tavolo. Il lavoro di Calleja (2010: 345) propone una visione più critica dei giochi rifiutando l'assunto di escapismo. Infine, non avendo incontrato processi discriminatori legati a nazionalità, colore della pelle, abilità, religione e classe sociale non è stato possibile trarre conclusioni in merito. Infine, la presente ricerca non pretende di essere esaustiva o rappresentativa di tutti i contesti di game sharing italiani, sono presenti dinamiche specifiche in ogni regione legate a variabili storico culturali del mondo di gioco in Italia. Lo studio vuole porsi come un ulteriore passo da aggiungere a una letteratura di ludologia attualmente in crescita in Italia, sviluppata per studiare e comprendere il gioco e le sue comunità. 


\section{Conclusioni} successo nel territorio italiano, dove da diversi anni si sta sviluppando un sempre maggiore interesse verso le potenzialità del gioco in contesti interpersonali, didattici e sociali. Come discusso precedentemente, questa pratica di sharing permette a chi ne prende parte di avere accesso a una rete sociale di saperi, competenze e relazioni significative basate su una passione che, se ben coltivata, offre potenzialità di crescita personale e apprendimento. La ricerca ha voluto dare spazio alle domande di riconoscimento dei contesti e delle reti di gioco, ovvero il volersi affermare come attività valida per tutte le età e forme di tempo libero. Il tempo libero stesso non è un fenomeno opposto al lavoro, come invece viene spesso trattato in letteratura, ma un insieme di spazi, tempi e atteggiamenti che possiede un valore intrinseco che va indagato indipendentemente (Karpatschof 2013). L'approccio etnografico della ricerca ha fatto emergere come vengono generati repertori culturali legati al tempo libero e come si intrecciano anche con dinamiche socialmente rilevanti come i rapporti tra generi e l'orientamento sessuale. Come sostenuto da Boellstorff (2006: 32), l'osservazione partecipante è un metodo estremamente adatto a studiare la cultura di gioco e l'attività ludica, che viene presa molto seriamente da chi la rende attiva e la promuove. A partire dalle diverse motivazioni che chi gioca porta al tavolo e gli stili di gioco (competitivi o cooperativi), queste forme di tempo libero si basano sulla condivisione di momenti significativi legati a una passione, regolata da norme formali e informali di grande interesse per le scienze sociali.

Per quanto riguarda gli aspetti educativi, il recente successo di approcci didattici basati sul gioco (Mochocki 2013: 57) potrebbe portare a un numero maggiore di ricerche sia sugli effetti sull'apprendimento che su altre variabili psicosociali rilevanti (Graham et al. 2008: 39; Fein et al. 2015: 306; Khan et al. 2015: 197). Le modalità di gamification come processo di apprendimento tramite strumenti simili al gioco hanno in realtà attirato le critiche (Lieberoth et al. 2015: 3) di molti giocatori ed esperti di settore ludici, i quali sostengono, e hanno dimostrato, che implementando meccaniche e processi di gioco "profondi" è possibile ottenere risultati, migliori e più efficaci, nelle relazioni sociali $\mathrm{e}$ nell'apprendimento (Mochocki 2013: 57). Le ambientazioni e i contenuti dei giochi sono spesso state utilizzate per trattare tematiche rilevanti dal punto di vista sociale, per promuovere riflessioni ecologiste (Reckien et al. 2013: 254), per sviluppare una maggiore sensibilità culturale (Ong-Flahertry et al. 2017).

In conclusione, le reti sociali di gioco si mostrano durature nel tempo e in continua espansione nei contesti familiari, stretti e allargati, grazie al loro potenziale di condivisione con le persone care, amici e familiari. Poiché il giocare sta acquisendo una rilevanza significativa nella vita delle persone, chi scrive ritiene che le scienze sociali possono trarre ottimi benefici da ricerche più consapevoli e partecipate sui giochi, online e offline, e non solo restare a guardare un mondo spesso considerato poco meritevole di essere giocato. 


\section{BIBLIOGRAFIA}

American Psychological Association, 2015 «Guidelines for psychological practice with transgender and gender nonconforming people», American Psychologist, 70(9): 832-864.

Arjoranta J., 2014 «Game Definitions: A Wittgensteinian Approach», Game Studies: the international journal of computer game research and Culture, 14(1), 29-35.

Baid H. et al., 2010 «Enjoyable learning: the role of humour, games, and fun activities in nursing and midwifery education», Nurse Education Today, 30(6): 548-552.

Bányai F. et al., 2018 «The psychology of esports: A systematic literature review», Journal of gambling studies, 1-15.

Belk R., 2009 «Sharing», Journal of consumer research, 36(5): 715-734.

Boellstorff T., 2006 «A ludicrous discipline? Ethnography and game studies», Games and Culture, 1(1), 29-35.

Bruner J.S., 1990 Acts of meaning (Vol. 3), Harvard University Press, Cambridge (MA).

Caillois R., 2001 Man, play, and games, University of Illinois Press, Champaign, trad. it., I Giochi e gli uomini: la maschera e la vertigine, a cura di L. Guarino, Giunti, Firenze, 2014.

Calleja G., 2010 «Digital games and escapism», Games and Culture, 5(4): 335-353.

Chen J., 2007 «Flow in games (and everything else)», Communications of the ACM, 50(4): 31-34.

Chick G., 2015 «Anthropology and the Study of Play», in J.E. Johnson, Eberle, S.G., Henricks T.S., Kuschner, D. (eds.), The handbook of the study of play (Vol. 2), Lanham, Rowman \& Littlefield: 71-84

Condis M., 2018 «Gaming Masculinity: Trolls, Fake Geeks, and the Gendered Battle for Online Culture», University of Iowa Press.

Crisp R. J. et al. 2009 «Can imagined interactions produce positive perceptions?: Reducing prejudice through simulated social contact», American psychologist, 64(4): 231.

Daniau S., 2016 «The Transformative Potential of Role-Playing Games: From Play Skills to Human Skills», Simulation \& Gaming, 47(4): 423-444.

Egenfeldt-Nielsen S., 2006 «Overview of research on the educational use of video games», Nordic Journal of Digital Literacy, 1 (03): 184-214.

Fein E., 2015 «Making meaningful worlds: role-playing subcultures and the autism spectrum», Culture, Medicine, and Psychiatry, 39(2): 299-321.

Gee J. P., 2003 «What video games have to teach us about learning and literacy», Computers in Entertainment (CIE), 1(1): 20-20.

Geertz C., 2000 Deep play: Notes on the Balinese cockfight», in L. Crothers, Lockhart C. (eds), Culture and Politics, Palgrave Macmillan, New York: 175-201.

Graham I. et al., 2008 «Experiential gaming to facilitate cultural awareness: its implication for developing emotional caring in nursing», Learning in Health and Social Care, 7(1): 37-45.

Gray K. L. et al., 2017 «Blurring the boundaries: Using Gamergate to examine "real" and symbolic violence against women in contemporary gaming culture», Sociology Compass, 11(3): e12458.

Huizinga J., 2002 Homo Ludens, Einaudi, Torino, 2002. 
Kapell M. W., 2015 The Play Versus Story Divide in Game Studies: Critical Essays, McFarland, Jefferson. Karpatschof B., 2013 «Play, but not simply play: The anthropology of play», in I. Schousboe, Winther-Lindqvist, D. (eds) Children's Play and Development, Dordrecht, Springer: 251-265.

Khan A. et al., 2015 «A study into the effects of a board game on flow in undergraduate business students», The International Journal of Management Education, 13(3): 193-201.

Lafreniere, M. A. et al., 2009 «On the costs and benefits of gaming: The role of passion», CyberPsychology \& Behavior, 12(3): 285-290.

Leone G. et al., 2013 La psicologia sociale: processi mentali, comunicazione e cultura, GLF editori Laterza, Bari.

Lieberoth A., 2015 «Shallow gamification: Testing psychological effects of framing an activity as a game», Games and Culture, 10(3), 229-248.

Lunghi C., 2017 «Reti collaborative di produzione e consumo», in L. Bovone, Lunghi C. (eds), Resistere. Innovazione e vita quotidiana, Donzelli editore, Roma: 65-90.

Mantovani G., 1998 L'elefante invisibile: tra negazione e affermazione delle diversità: scontri e incontri multiculturali, Giunti, Firenze.

Markey P.M. et al., 2015 «Violent video games and real-world violence: Rhetoric versus data», Psychology of Popular Media Culture, 4(4): 277.

Mochocki M., 2013 «Edu-larp as revision of subject-matter knowledge», International Journal of Role-Playing, 4: 55-75.

O'Connor K. J. et al., 2015 «Handbook of play therapy», John Wiley \& Sons, New Jersey: Hoboken.

Ong-Flaherty C. et al., 2017 «Effectiveness of gaming in creating cultural awareness», Learning, Culture and Social Interaction, 12: 149-158.

Oren A., 2008 "The use of board games in child psychotherapy», Journal of child psychotherapy, 34(3): 364-383.

Paolicchi P., 2002 «L'intervista narrativa in psicologia sociale», in B.M. Mazzara (ed), Metodi qualitativi in psicologia sociale, Roma, Carocci: 193-205.

Pieroni O.I. et al., 2000 «Classroom innovation: games to make chemistry more interesting and fun», The Chemical Educator, 5(4):167-170.

Reckien D. et al., 2013 «Climate change gaming on board and screen: A review», Simulation \& Gaming, 44(2-3): 253-271.

Schor J.B., 2014 Born to buy: The commercialized child and the new consumer cult, Simon and Schuster, New York.

Simon W. et al., 2003 «Sexual scripts: Origins, influences and changes», Qualitative sociology, 26(4): 491-497.

Turner V.W., 1982 From ritual to theatre: The Human seriousness of play, Paj Publications.

Williams D. et al., 2009 «The virtual census: Representations of gender, race and age in video games», New Media \& Society, 11(5): 815-834.

Wittgstein L., 1967 Ricerche Filosofiche, Einaudi, Torino.

Zichermann G. et al., 2013 Gamification Revolution, McGraw-Hill Education, New York. 
Zagal J.P. et al., 2006 «Collaborative games: Lessons learned from board games», Simulation \& Gaming, 37(1): 24-40.

Zagal J. P., 2010 Ludoliteracy: defining understanding and supporting games education, ETC Press, Halifax.

\section{NOTE}

1. In un suo articolo, nel definire il gioco seguendo l'approccio di Wittgenstein, Arjoranta (2014: 7) riflette su come i game studies possono beneficiare dai lavori di Wittgenstein ma bisogna tenere a mente che era un filosofo del linguaggio e non uno studioso di giochi.

2. Nella versione originale di questo dilemma etico sviluppato da Philippa Ruth Foot, un conducente di tram si trova su un carrello non in grado di frenare ma solo di cambiare rotaia tramite deviatoio. Sul binario dove si sta dirigendo il tram si trovano cinque persone legate e impossibilitate a muoversi. Tra il tram e queste cinque persone legate è presente un secondo binario parallelo con una singola persona legata, anch'essa impossibilitata a muoversi. Chi si trova vicino al deviatoio, ruolo interpretato dal rispondente, può scegliere tra due sole alternative: lasciar proseguire il tram uccidendo cinque persone, oppure fargli cambiare rotaia e fare in modo che il tram ne uccida una sola.

3. Un termine nato come dispregiativo per indicare una persona con una predisposizione per la tecnologia, attività intellettuali e ridotta propensione alla socializzazione, successivamente rivendicato con orgoglio (Condis 2018).

4. Nel contesto del gioco, la presenza delle donne al tavolo è spesso vista come un premio che viene loro concesso, una "quota rosa" (Gray et al. 2017). Questo atteggiamento rimarca l'idea maschile che vede nelle donne soggetti inadatti ai giochi di ruolo o da tavolo, la cui presenza è tollerata nella misura in cui permette alla componente maschile di godere di una presenza simile a quella di una valletta.

5. Con playtest si intende il processo di collaudo e valutazione di un gioco o un'attività per identificare difetti, bug o problemi di design prima di rendere accessibile un prodotto; i playtest possono essere aperti al pubblico, chiusi o con altre caratteristiche. Questo processo è molto comune nel design di videogiochi, giochi da tavolo e giochi di ruolo ed è diventato nel tempo fondamentale per valutare sia la qualità di un prodotto sia per comprendere se chi lo sviluppa tiene alle opinioni di chi lo acquisterebbe e dei fan (fanbase).

6. Un aggettivo utilizzato per descrivere una persona la cui identità di genere ed espressione di genere si allinea con il sesso assegnato alla nascita (APA, 2015).

\section{RIASSUNTI}

Il gioco occupa per molte persone una parte sostanziale del proprio tempo libero ed è diventata un'occasione per creare reti di socialità, spazi di partecipazione, identità ma anche occasioni di consumo e condivisione. Grazie al diffondersi e al consolidarsi delle culture di gioco sempre più spazi di condivisione di giochi si fanno strada in Italia. Questa pratica, denominata game sharing, porta singole persone a creare gruppi di gioco e associazioni per acquistare in gruppo giochi da tavolo e di ruolo da condividere sia tra di loro che con chi inizia a giocare, il tutto seguendo le 
logiche no-profit, la passione per i giochi e il fare community. Il presente contributo offre in primis una breve riflessione sul concetto e lo studio del gioco nella scienze sociali e, inoltre, presenta una ricerca qualitativa tra antropologia e psicologia sociale condotta in diverse città italiane (Bologna, Catania, Padova, Torino e Treviso). Nel corso delle ricerca, sono state condotte multiple osservazioni partecipanti di associazioni di game sharing, di negozi che attuano questa pratica e di gruppi di gioco indipendenti. Sono state inoltre svolte delle interviste narrative con organizzatori e coordinatori di associazioni, con negozianti, creatori di giochi e, infine, con giocatori e giocatrici che usano il gioco per fare attivismo su tematiche di genere e orientamento sessuale. Il contributo indaga i contesti di gioco come rete sociale dedicata al tempo libero e alla condivisione, come attività di apprendimento non formale di empatia e skill sociali e, infine, come una rete di saperi professionali a cui ricorrere in caso di bisogno.

To many people playing is a substantial part of their free time and has become an opportunity to create societal networks, spaces for participation, identity but also opportunities for consumption and sharing. Thanks to the spreading and the consolidation of game cultures, more and more spaces for sharing games have found their way into Italy. This practice, called 'game sharing', leads individuals to create groups of gamers and associations to collectively buy board and roleplaying games to share with each other and with brand-new players. This practices follows a non-profit logic and arise from the passion for games and community. The present contribution offers in the first part a brief summary on the concepts and the study of the games in social sciences whereas, in the second part, presents a qualitative research between anthropology and social psychology conducted in different Italian cities (Bologna, Catania, Padua, Turin and Treviso). The research included multiple participatory observations of game sharing associations, stores that implemented this practice and independent game groups. In addition to ethnographic observation, many narrative interviews were conducted with organizers and coordinators of associations, with shop owners, game designers and, finally, with players who use games and roleplay to do activism on gender issues and sexual orientation. The contribution investigates gaming contexts as a societal network dedicated to free time and sharing, as a non-formal learning activity of social skills and empathy and, finally, as a network of professional knowledge to be used in case of need.

\section{INDICE}

Keywords : game sharing, informal learning, sharing, gamification, free time

Parole chiave : game sharing, apprendimento non formale, sharing, gamification, tempo libero

\section{AUTORE}

\section{AURELIO CASTRO}

Dipartimento di Filosofia, Sociologia, Pedagogia e Psicologia Applicata (FISPPA), Università degli Studi di Padova aurelio.castro@phd.unipd.it 